\title{
PLASMAS COLLISIONNELS
}

Physique des décharges RF et MicRO-ONDE 


\section{Grenoble Sciences}

Grenoble Sciences est un centre de conseil, expertise et labellisation de l'enseignement supérieur français. Il expertise les projets scientifiques des auteurs dans une démarche à plusieurs niveaux (référés anonymes, comité de lecture interactif) qui permet la labellisation des meilleurs projets après leur optimisation. Les ouvrages labellisés dans une collection de Grenoble Sciences ou portant la mention « Sélectionné par Grenoble Sciences » (Selected by Grenoble Sciences) correspondent à :

- des projets clairement définis sans contrainte de mode ou de programme,

- des qualités scientifiques et pédagogiques certifiées par le mode de sélection (les membres du comité de lecture interactif sont cités au début de l'ouvrage),

- une qualité de réalisation assurée par le centre technique de Grenoble Sciences.

\section{Directeur scientifique de Grenoble Sciences}

Jean Bornarel, Professeur émérite à l'Université Joseph Fourier, Grenoble 1

Pour mieux connaître Grenoble Sciences :

https://grenoble-sciences.ujf-grenoble.fr

Pour contacter Grenoble Sciences :

Tél : (33) 4765146 95, e-mail : grenoble.sciences@ujf-grenoble.fr

\section{Livres et pap-ebooks}

Grenoble Sciences labellise des livres papier (en langue française et en langue anglaise) mais également des ouvrages utilisant d'autres supports. Dans ce contexte, situons le concept de pap-ebook. Celui-ci se compose de deux éléments :

- un livre papier qui demeure l'objet central avec toutes les qualités que l'on connaît au livre papier,

- un site web compagnon qui propose :

> des éléments permettant de combler les lacunes du lecteur qui ne possèderait pas les prérequis nécessaires à une utilisation optimale de l'ouvrage,

$>$ des exercices pour s'entraîner,

$>$ des compléments pour approfondir un thème, trouver des liens sur internet, etc.

Le livre du pap-ebook est autosuffisant et certains lecteurs n'utiliseront pas le site web compagnon. D'autres l'utiliseront et ce, chacun à sa manière. Un livre qui fait partie d'un pap-ebook porte en première de couverture un logo caractéristique et le lecteur trouvera la liste de nos sites compagnons à l'adresse internet suivante :

https://grenoble-sciences.ujf-grenoble.fr/pap-ebooks

Grenoble Sciences bénéficie du soutien du ministère de l'Enseignement supérieur et de la Recherche et de la région Rhône-Alpes.

Grenoble Sciences est rattaché à l'Université Joseph Fourier de Grenoble.

ISBN 9782759811298

(C) EDP Sciences 2014 


\section{PLASMAS COLLISIONNELS \\ Physique des décharges RF eT MICRO-ONDE}

Michel Moisan et Jacques Pelletier

\section{edpsciences}

17, avenue du Hoggar

Parc d'Activité de Courtabœuf - BP 112

91944 Les Ulis Cedex A - France 


\section{Plasmas collisionnels \\ Physique des décharges RF et micro-onde}

Cet ouvrage, labellisé par Grenoble Sciences, est un des titres du secteur Sciences de la Matière de la collection Grenoble Sciences d'EDP Sciences, qui regroupe des projets originaux et de qualité. Cette collection est dirigée par Jean BORNAREL, Professeur émérite à l'Université Joseph Fourier, Grenoble 1.

\section{Comité de lecture de la première édition :}

- Michel Aubès, Professeur à l'Université Paul Sabatier (Toulouse)

- Jacques Derouard, Professeur à l'Université Joseph Fourier (Grenoble)

- Ana Lacoste, Professeur à l'Université Joseph Fourier (Grenoble)

- Bachir SAOUdi, physicien à l'Université de Montréal

- avec le concours de Cédric DE VAULX et Didier RIEU

Mise en page : Danielle KéROACK ; figures : Danielle KérOACK et Sylvie Bordage ; illustration de couverture : Alice GIRAUD, d'après les photos fournies par Ana LACOSTE (Université Joseph Fourier). Cette nouvelle édition a été suivie par Stéphanie TRINE, Anne-Laure Passavant et Sylvie Bordage.

\section{Autres ouvrages labellisés sur des thèmes proches (chez le même éditeur) :}

Introduction à la mécanique statistique $(E$. Belorizky \& W. Gorecki) • Mécanique statistique. Exercices et problèmes corrigés $(E$. Belorizky \& W. Gorecki) $\bullet$ La cavitation. Mécanismes physiques et aspects industriels (J.P. Franc et al.) • Outils mathématiques à l'usage des scientifiques et ingénieurs (E. Belorizky) - Magnétisme : I Fondements, II Matériaux (sous la direction d'E. du Trémolet de Lacheisserie) $\bullet$ Physique des diélectriques (D. Gignoux \& J.C. Peuzin $)$ Supraconductivité. Introduction $($ P. Mangin \& R. Khan $) \cdot$ Spectroscopie de résonance paramagnétique électronique. Fondements $(P$. Bertrand $) \bullet$ Spectroscopies infrarouge et Raman (R. Poilblanc \& F. Crasnier) $\bullet$ La mécanique quantique. Problèmes résolus, Tome I et II (V.M. Galitski, B.M. Karnakov \& V.I. Kogan) • L'air et l'eau (R. Moreau) • Turbulence (M. Lesieur) • Les milieux aérosols et leurs représentations (A. Mailliat) • Mécanique. De la formulation lagrangienne au chaos hamiltonien $($ C. Gignoux $\&$ B. Silvestre-Brac $) \cdot$ Description de la symétrie. Des groupes de symétrie aux structures fractales (J. Sivardière) $\bullet$ Symétrie et propriétés physiques. Des principes de Curie aux brisures de symétrie (J. Sivardière) • Energie et environnement. Les risques et les enjeux d'une crise annoncée (B. Durand) $\bullet$ En Physique, pour comprendre (L. Viennot) • Naissance de la Physique (M. Soutif) $\bullet$ Du Soleil à la Terre. Aéronomie et météorologie de l'espace (J. Lilensten \& P.L. Blelly) • Sous les feux du Soleil, vers une météorologie de l'espace (J. Lilensten \& J. Bornarel) • Méthodes numériques appliquées (J.P. Grivet) • Analyse numérique et équations différentielles (J.P. Demailly) • Analyse statistique des données expérimentales (K. Protassov) • Minimum Competence in Scientific English (J. Upjohn, S. Blattes \& V. Jans) • Approximation hilbertienne (J. Gaches \& M. Attéia)

et d'autres titres sur le site internet :

https://grenoble-sciences.ujf-grenoble.fr 